\title{
Evaluation of Blood Stream Infections by Candida in Three Tertiary Hospitals in Salvador, Brazil: A Case-Control Study
}

\author{
Maria Goreth Barberino ${ }^{1}$, Nanci Silva ${ }^{2}$, \\ Carla Rebouçass ${ }^{3}$, Katarine Barreiro, ${ }^{1}$, \\ Ana Paula Alcântara ${ }^{4}$, Eduardo M. Netto ${ }^{3}$, \\ Lígia Albuquerque $^{3}$ and Carlos Brites ${ }^{2,3}$
}

\author{
${ }^{1}$ Aliança Hospital, ${ }^{2}$ Espanhol Hospital, ${ }^{3}$ Federal University of \\ Bahia, ${ }^{4}$ Santo Amaro Hospital; Salvador, BA, Brazil
}

\begin{abstract}
Invasive infections caused by Candida spp. are an important problem in immunocompromised patients. There is scarce data on the epidemiology of blood stream candidiasis in Salvador, Brazil. This study evaluates the risk factors associated with candidemia, among patients admitted to three tertiary, private hospitals, in Salvador, Brazil. We conducted a case-control, retrospective study to compare patients with diagnosis of candidemia in three different tertiary hospitals in Salvador, Brazil. Patients were matched for nosocomial, acquired infections, according to the causal agent: cases were defined by positive blood cultures for Candida species. Controls were those patients who had a diagnosis of systemic bacterial infection, with a positive blood culture to any bacteria, within the same time period ( $\pm \mathbf{3 0}$ days) of case identification. The groups were compared for the main known risk factors for candidemia and for mortality rates. A hundred thirty-eight patients were identified. Among the 69 cases, only 14 were diagnosed as infected by Candida albicans. Candida species were defined in only eight cultures: $C$. tropicalis (4 cases), $C$. glabrata, $C$. parapsilosis, $C$. guillermondi, $C$. formata ( 1 case each). The main risk factors, identified in a univariate analysis, were: presence of a central venous catheter (CVC), use of parenteral nutrition support (PNS), previous exposure to antibiotics, and chronic renal failure (CRF). No association was detected with surgical procedures, diabetes mellitus, neutropenia or malignancies. Patients were more likely to die during the hospitalization period, but the rates of death caused by the infections were similar for cases and controls. The length of hospitalization was similar for both groups, as well as the time for a positive blood culture. Blood stream infection by Candida spp. is associated with CVC, PNS, previous use of antibiotics, and CRF. The higher mortality rate for cases probably better reflects the severity of the underlying diseases, than as a direct consequence of Candidemia.

Key Words: Invasive candidiasis, risk factors, bacteremia.
\end{abstract}

Candida species are the most common cause of fungal infections worldwide. They can cause a great variety of infections, including simple, mucocutaneous processes, but they also provoke severe, invasive infections that can involve virtually any organ. Blood stream infections by Candida are increasingly common, and often are associated with high mortality rates [1,2]. Recently, we have seen an important increment in the frequency of non-albicans species of Candida, such as C. glabrata, C. krusei, C. tropicalis, and C. parapsilosis, as causes of fungemia [3,4].

Invasive candidiasis is a severe, life-threatening infection, with a mortality rate comparable to that observed in septic shock patients (40\%-60\%), and it occurs mostly in immunosuppressed patients. This is particularly true for bone marrow transplant recipients, and those under chemotherapy, in which the mortality rate is as high as $80 \%$.

Received on 17 September 2005; revised 23 January 2006. Address for correspondence:Dr. Nanci Silva. Av. Juracy Magalhães jr, 2096, sl. 601, Rio Vermelho, Salvador, Bahia, Brazil. Phone:-5571-21084640.Fax-55-71-34522950.E-mail: silva.nanci@uol.com.br

The Brazilian Journal of Infectious Diseases 2006;10(1):36-40. (C) 2006 by The Brazilian Journal of Infectious Diseases and Contexto Publishing. All rights reserved.
Candida species causing invasive infections seem to vary according to the world region. Risk factors, sensitivity pattern, and outcomes can differ widely, depending on the region we analyze [5,6]. Colombo, in 2003, published the results of a multicenter, randomized study, which included 239 adult patients, from 20 countries, in five continents. Candida albicans was the most frequently isolated species in all regions, accounting for $45 \%$ of the total [7]. However, non-albicans species were more common. In the USA, $C$. glabrata was the second most commonly isolated species. In contrast, $C$. parapsilosis and C. tropicalis were responsible for $55 \%$ of cases in Latin America.

The first well-documented case of deep organ infection (brain) by Candida was described by Zenker, in 1861 [8]. The first report of disseminated candidiasis in an adult patient was published in 1937, by Bogden and Kessel [9]. In 1940, endocarditis by Candida was also reported for the first time [10]. A review of such cases, performed by Hurley, in 1964 detected 16 reports on disseminated candidiasis [11]. The reports on Candida isolation from the blood stream became more frequent after the introduction of antibiotics use as a usual practice, in 1940 [12].

Nowadays, Candida spp. is known as the $4^{\text {th }}$ most frequently isolated pathogen from the blood stream, among hospitalized patients in North American hospitals [13,14]. 
Disseminated candidiasis is a consequence of medical progress. Invasive fungal infections are more prevalent due to the increasing number of high-risk patients, with different degrees of immunosuppression. Underlying diseases or chronic conditions, like cancer, bone marrow transplantation, AIDS, and chronic use of corticosteroids, are risk factors for fungal infections, and surgical procedures, extended use of catheters, and extended spectrum antibiotics are frequently associated with severe fungal diseases [15].

Candida spp. were rarely identified before 1960 . However, these pathogens are now recognized as among the most important pathogens causing nosocomial infections, according to the National Nosocomial Infections Surveillance System [16]. They are one of the leading causes of blood stream infections, with a mortality rate of $40 \%$. The incidence of such infections nearly doubled from 2.0 to 3.8/1000 patients discharged in American hospitals [17]. The main increase occurred in internal medicine and surgical clinics, but not in oncology or transplantation units. This suggests that fungal infections are no longer limited to immunosuppressed patients [18].

The reasons for this increase in fungal infections are multifactorial: better clinical evaluation, and diagnosis, greater survival for patients with malignancies, chronic diseases, increasing number of transplants, complex surgical procedures, catheters, implants and use of wide spectrum antibiotics [19]. There is little information on Candida infections in Bahia. We designed this study to evaluate the risk factors and outcomes of patients presenting with blood stream Candida infections in three private hospitals in Salvador, Brazil.

\section{Material and Methods}

Study design. This was a case-control, retrospective, multicentric study to evaluate risk factors, clinical characteristics and outcomes for patients who had Candida spp. isolated from the blood stream in three different private hospitals, in Salvador, Bahia, Brazil. Cases were defined as patients with a blood stream, hospital acquired infection caused by Candida spp., in the last eight years. Controls were defined as patients admitted at the same period of time as the cases (or within a three week interval), who had a diagnosis of nosocomial bacterial blood stream infection. We recorded data on age, gender, time of hospitalization before infection diagnosis, previous use of antibiotics and its duration, underlying disease, and final outcomes.

Settings. The study was conducted at three different hospitals in Salvador. The first one, Hospital Espanhol (HE) is a tertiary, private, 300 bed-hospital. The HE has a 25 bed Intensive Care Unit (ICU), and a neonatal ICU with 12 beds. The second hospital, Hospital Aliança (HA), is also a private, tertiary care, 160 bed-hospital, with a 15 bed general ICU, and a 6 bed cardiac ICU. The third institution is Hospital Santo Amaro (HAS), a tertiary, private, 150 bed hospital, with a 5 bed ICU and a neonatal ICU with 15 beds.

Data collection. We reviewed all laboratory reports from the three hospitals made during the previous eight years. The positive blood stream cultures for Candida spp. were recorded, and these cases were paired with other presenting bacterial infections at the same site, within a three-week interval of Candida isolation. The data were stored in a database, using the software SPSS for windows, version 12.0. The statistical analysis was also performed with this computer program.

\section{Results}

A total of 138 patients were enrolled in the study (69 cases). The distribution by hospital showed a greater number of enrolled patients at HA (58), followed by HAS, and HE (40 patients each). The gender frequency was identical for cases and controls (50\% each). Mean age in years was also similar for cases $(51.6 \pm 31.9)$ and controls $(53.4 \pm 29.2, \mathrm{p}=0.1)$. The number of positive blood cultures was also similar for both groups, although we have found a trend for a greater number of positive blood cultures for cases, when compared to controls (73\% of cases had three positive blood cultures compared with only $27 \%$ of controls, $p=0.08$, chi-square test). Also, there was a trend towards more frequent isolation of Candida among patients in ICUs (62.6\%), than blood stream bacterial infection $(49.2 \%$, odds ratios $(\mathrm{OR})=1.79 ; 95 \%$ confidence interval (CI): $0.87-3.4, \mathrm{p}=0.08$, Fisher exact test).

The identification of Candida species was only available for 25 samples: 14 were classified as Candida albicans, 4 as C. tropicalis, and 3 as non-albicans species (without identification). Candida glabrata, C. parapsilosis, C. formata and $C$. guilliermondi were identified in one culture each. Fortyfour cultures had the final resulted released as Candida spp. Table 1 shows the frequency of bacterial infection identified among the controls.

Surgical procedures were not associated with Candida infection: cases were more likely to report a previous surgical procedure $(55.7 \%)$ than controls $(42.4 \%)$, but this difference was not significant (relative risk $(\mathrm{RR})=1.71$; $95 \% \mathrm{CI}$ : 0.82 $3.58, \mathrm{p}=0.16$ ). In addition, report of previous use of antibiotics was significantly associated with Candida infection: it was reported by $58.4 \%$ of cases and by only $41.6 \%$ of the controls $(\mathrm{RR}=4.91 ; 95 \% \mathrm{CI}=0.88-35.99$, one-sided $\mathrm{p}=0.03)$. Nevertheless, the duration of antibiotic use was similar for both groups (13.4 versus 15.9 days, for cases and controls, respectively, $\mathrm{p}=0.5$ ) as was the number of drugs used by each group of patients.

Another risk significantly associated with Candida infection was use of central venous catheters (CVC). The frequency of that device use was $72 \%$ for cases, against $56 \%$ for controls ( $\mathrm{OR}=2.0$; 95\% CI: $0.99-4.12$, one-sided $\mathrm{P}$ value: 0.037 , by the Fisher exact test). In addition, the use of parenteral 
Table 1. Frequency of bacterial agents isolated among controls

\begin{tabular}{|c|c|}
\hline Bacteria & $\begin{array}{c}\text { Number of positive } \\
\text { cultures }\end{array}$ \\
\hline Acinetobacter baumannii/haemolytic & 1 \\
\hline Acinetobacter baumannii & 1 \\
\hline E. aerogenes & 1 \\
\hline E. aglomerans & 1 \\
\hline E. gergoviae & 1 \\
\hline Klebsiella oxytoca & 1 \\
\hline Ralstonia piccketti & 1 \\
\hline S. agalactiae & 1 \\
\hline S. viridans & 1 \\
\hline Serratia marcescens & 1 \\
\hline Staphilococcus hominis & 1 \\
\hline Staphylococcus auricularis & 1 \\
\hline Staphylococcus sp. & 1 \\
\hline Streptococcus alfa-hemolytic & 1 \\
\hline Streptococcus sp. & 1 \\
\hline P. fluorescens/putida & 2 \\
\hline Pseudomonas fluorescens & 2 \\
\hline S. haemolyticus & 2 \\
\hline Acinetobacter linoffii & 3 \\
\hline Enterobacter cloacae & 3 \\
\hline S. hominis & 3 \\
\hline Stenotrophomonas maltophilia & 3 \\
\hline E. coli & 6 \\
\hline K. pneumoniae & 6 \\
\hline S. epidermidis & 6 \\
\hline P. aeruginosa & 8 \\
\hline S. aureus & 10 \\
\hline Total & 69 \\
\hline
\end{tabular}

nutrition (PN) was also strongly predictive of Candida infection (OR=3.3; 95\% CI: $1.5-7.1, \mathrm{p}=0.02)$. An association was also detected between fungal infection and use of corticosteroids (OR: 1.55 ; 95\% CI: $1.08-2.22, \mathrm{p}=0.049$ ), and for patients with diagnosis of chronic renal failure $(\mathrm{OR}=1.95$; $95 \%$ CI: $1.5-2.5, p=0.004)$. On the other hand, diagnosis of malignancies, neutropenia and diabetes mellitus, as the underlying disease, were not associated with development of Candida infection. Table 2 displays the main risk factors and their association with Candidemia.

A consistent association was detected between candidemia and death during the hospitalization period: patients presenting blood stream infection by Candida were more likely to die during hospitalization (OR=2.24; $95 \%$ CI: $1.05-4.81$, $\mathrm{p}=0.03$ ). However, this tendency was not detected when we analyzed only the deaths directly attributable to the infections $(\mathrm{OR}=2.4$; $95 \%$ CI: $0.48-12.1, \mathrm{p}=0.2)$.

The mean hospitalization period in days was similar for cases and controls $(73.2 \pm 7.1$ versus $61.2 \pm 8.1, \mathrm{p}=0.3)$. The time in days till detection of fungemia (cases) after hospital admission was longer $(32.6 \pm 26.7)$ than that for detection of bacteremia among controls $(26.4 \pm 38.9)$, but the difference was not significant.

We performed a multivariate analysis, including all significant risk factors detected in the preliminary evaluation, but the resulting model showed considerable overlapping of the variables associated with candidemia. This fact generated an unstable model, which could not identify any single significant risk factor, due to the low number of samples in the

Table 2. Risk factor for invasive Candidiasis in three different hospitals, in Bahia, Brazil

\begin{tabular}{lcccc}
\hline Risk factor & Cases N $(\%)$ & Controls N (\%) & OR (95\% CI) & p value \\
\hline Gender & 40 & & & \\
$\quad$ Male & 29 & $40-$ & NS & \\
Female & $73,2 \pm 58.8$ & $61.1 \pm 67.0$ & NS & \\
Mean duration of Hospitalization (days \pm sd) & $32.7 \pm 26.7$ & $26.4 \pm 38.9$ & NS & \\
Time in hospital before a positive culture & $58(84.1)$ & $44(63.8)$ & $4.91(0.88-35.99)$ & 0.03 (one sided) \\
Previous use of antibiotics & $30(43.4)$ & $13(18.8)$ & $3.31(1.53-7.14)$ & \\
Parenteral Nutrition & $44(63.8)$ & $35(50.7)$ & $1.71(0.87-3.38)$ & 0.08 \\
Previous surgery & $50(72.4)$ & $39(56.5)$ & $2.0(0.99-4.12)$ & 0.037 \\
CVC & $11(15.9)$ & $4(5.8)$ & $1.56(1.09-2.22)$ & 0.049 \\
Use of corticosteroids & $0(0)$ & $3(4.3)$ & NS & \\
Neutropenia & $10(14.5)$ & $4(5.8)$ & $0.56(0.23-1.34)$ & NS \\
Malignancies & $10(14.5)$ & $1(1.5)$ & $11.5(1.43-92.71)$ & 0.004 \\
Chronic renal failure & $12(17.4)$ & $12(17.4)$ & $1.0(0.41-2.41)$ & NS \\
Diabetes mellitus & $34(49.3)$ & $21(30.4)$ & $2.24(1.05-4.80)$ & 0.03 \\
Death & $29(42.0)$ & $16(23.9)$ & $2.42(0.39-16.02)$ & NS \\
Death related to the infection & & & & \\
\hline
\end{tabular}


study, and the prevalence of multiple conditions associated with the main risks that were initially detected.

\section{Discussion}

Candida species are the most common causes of fungal infection. In Brazil, several reports have shown that nonalbicans species of Candida are more prevalent in tertiary hospitals [20]. In Bahia, there is no previous epidemiological information on the characteristics of Candidemia. Our report confirms some previously described findings from Brazilian hospitals, but it also reveals some important differences: We detected a predominance of non-albicans species in three tertiary hospitals, but the species definition was possible for only part of the patients. In Bahia, there is no specialized mycology laboratory, and the routine procedure is to classify the non-albicans species as Candida spp. Recently, we have seen some changes in this practice, with a greater effort of some private hospital laboratories to properly identify the species of Candida promoting infection in hospitalized patients. Since our work was performed in a retrospective manner, there was no chance to precisely discriminate albicans and non-albicans species of Candida, unless it had already been done during the isolation procedures. Nevertheless, we detected a large proportion of non-albicans species, (here reported as spp.), and we were able to identify the main risk factors associated with such infections.

In a similar way to that reported by various other authors, we found that candidemia was significantly associated with antibiotic use (but not with the duration of its use), presence of a central venous catheter, use of parenteral nutritional support, and chronic renal failure [21]. No association was detected with other recognized risks for invasive candidiasis, such as diabetes mellitus, neutropenia, and malignancies. The relatively low number of some of these conditions in our sample may explain such discrepancies (for instance, only three patients presented with neutropenia), but for the remaining, it could mean a real lack of association between such conditions and Candida infection in our hospitals. It could represent a distinctive pattern of risk for acquisition of invasive candidiasis in Bahia, or only a different at-risk population in our hospitals.

The results from our study reinforce the evidence for the already recognized risk of invasive candidemia in chronic renal failure patients. These individuals are often submitted to invasive procedures, are much more exposed to antibiotics than the general population (due to the higher risk of bacterial infections), and are considered to be immunocompromised patients. In addition, the need for maintenance of ready access to dyalitic procedures increases the risk for acquiring bacterial infections (blood stream infections, peritonitis, etc). The consequent greater use of antibiotics is a potential risk factor for the selection of resistant bacterial infections, and for the acquisition of fungal infections [22].
The same rationale may explain the higher risk found for patients using parenteral nutrition support. These individuals are frequently severely ill, and they are often affected by debilitating diseases, which increases the chance of use of antibiotics, invasive procedures, and the use of corticosteroids $[23,24]$. All patients with severe disease usually need a central vascular access line. Thus, the finding of $\mathrm{CVC}$ as a risk factor for candidiasis is also expected, and it may be related to the catheter itself, but, conversely, it could also be a marker of the severity of the underlying disease.

Candidiasis is a life-threatening infection, with mortality rates often reaching $50 \%$, or higher [25]. There is also some evidence suggesting that non-albicans species could be associated with a poorer prognosis. In our study, candidemia was associated with a higher mortality rate, but when we analyzed the mortality attributable to infection, there was no difference between cases and controls. This suggests that invasive candidiasis, in our study, was a better marker for disease severity, than an independent risk for mortality during the course of infection.

The choice of controls presenting with blood stream, nosocomial bacterial infection made it possible to avoid other potential confounding factors, since most of these patients had a similar diagnosis, a similar length of hospitalization, and similar time to acquire the blood stream infection. Also, the gender distribution and mean age were almost identical for both groups. This approach differs from other studies, where patients were compared to controls without candidemia, but not presenting nosocomial infections, which could lead to potential bias in the analysis of risk factors.

In conclusion, candidemia is associated with well-known risk factors in tertiary hospitals in Salvador, Brazil. There was a higher prevalence of non-albicans species, but the characterizations of these isolates was not performed, which does not allow us to define the exact profile of such infections in our hospitals. However, the finding of $C$. tropicalis in three of seven species-identified samples suggests that this species could be moere prevalent in Salvador.

\section{References}

1. Asmunds Dottir L.R., Erlends Dottir H., Gott Fredsson M. Increasing incidence of Candidemia: Results from a 20 - Year Nationwide study in Iceland. J Clin Microbiol 2002;40:348992.

2. Wise G.J., Silver D.A. Fungal Infections of the Genitourinary System. J Urol 1993;149:1377-88.

3. Fraser V.J., Jones M., Dunkel J., et al. Candidemia in Tertiary care Hospital: Epidemiology, Risk Factors and Predictors of mortality. Clin Infect Dis 1992;15:414-21.

4. Girmenia C., Martiwo P. Fluconazol and the Changing Epidemiology of candidemia. Clin Infect Dis 1998;27:232-4.

5. Rancel Frausto M.S., Wiblin T., et al. National Epidemiology of Mycosis Survey (Nemis): Variations in Rates of Candidal Bloodstream Infections in seven Surgical Icus and six Neonatal Icus. Clin Infect dis 1999;29:253-8. 
6. Pfaler M.A., Messer S.A., Houston A., et al. National Epidemiology of Mycoses Survey: A Multicenter Study of Strain Variation an Antifungal Susceptibility Among Isolates of Candida Species. Diagn Microbiol Infect Dis 1998;31:289-96.

7. Colombo A.L., Perfect J., Dinubile M., et al. Global Distribution and Outcomes for Candida Species causing invasive Candidiasis: Results from an International Randomized DoubleBlind Study of Caspofungin Versus Amphotericin B for the treatment of invasive Candidiasis. European journal of Clinical Microbiology and Infectious Diseases 2003;22:470-4.

8. Bodey G.P. Hematogenous and major organ candidiasis. In Candidiasis: pathogenesis, diagnosis and treatment, Raven Press Ltd., New York, 1993.

9. Bodgen E., Kessel J. Monilial Meningitis. Arch Pathol 1937;23:909-12.

10. Joachim H., Polayes S. Aubacute Endocarditis and Systemic Mycosis (Monilia). Jama 1940;115:205-2004.

11. Hurley R. Acute Disseminated Septicemic Moniliasis in adults an children. Postgrad Med J 1964;40:644-53.

12. Edwards J.R. Jr. Invasive Candida Infections. Evolution of a Fungal Pathogen. N Engl J Med 1991;324:1060-2.

13. Fridkin S.K., Jarvis W.R. Epidemiology of Nososcomial Funcal Infections. Clin Microbiol Rev 1996;9:499-511.

14. Wey S.B., Mori M., Pfaller M.A., et al. Hospital-Acquired Candidemia. The Attributable Mortality and Excess Length of Stay. Archives of Internal Medicine 1998;48:2642-5.

15. Beck-Sague C., Jarvis W.R., National Nosocomial Infections Surveillance System. Secular Trends in the Epidemiology of Nosocomial Fungal Infections in the United States, 19801990. Journal of Infectious Diseases 1993;167:1247-51.
16. Banerjee S.N., Emori T.G., Culver D.H., et al. Secular Trends in Nosocomial Primary Bloodstream Infections in the United States, 1980-1989. National Nosocomial Infections Surveillance System. American Journal of Medicine 1991;91:865-95.

17. Vazquez J.A., Sanchez V., Dembry L.M., et al. Nosomial Acquisition of Candida Albicans: An Epidemiologic Study. J Infect Dis 1993; 168:195-201.

18. Bross J., Talbot G.H., Maislin G., et al. Risk Factors for Nosocomial Candidemia: A Case-Control Study in adults Without leukemia. Am J Med 1989;87:61-4.

19. Colombo A.L., Nucci M., Salomão R., et al. High Rate of Non-Albicans Candidemia in Brazilian Tertiary Care Hospitals. Diagnostic Microbiology and Infections Diseases 1999;34:281-6.

20. Karabinis A., Hill C., Leclerca B., et al. A Risk factors for Candidemia in cancer patients: A Case-Control Study. J Clin Microbiol 1988;26:429-32.

21. Nucci M., Colombo A.L. Risk Factors for Breakthrough Candidemia. EUR J Clin Microbiol Infect Dis 2002;21:209-11.

22. Botas C.M., Kurlat J., Young S.M., Sola A. Disseminated Candidal Infectious and Intravenous Hydrocortisone in Preterm Infants. Pediatrics 1995;95:883-7.

23. Abi-Said D., Anaissie E., Uzun O., et al. the Epidemiology of Hematogenous Candidiasis By Different Candida Species. Clin Infect dis 1997;24:1122-8.

24. Blot S.I., Vandewoude K.H., Hoste E.A., Colardyn F.A. Effets of nosocomial Candidemia on outcomes of Critically Ill Patients. Am J Med 2002;113;480-5. 